\title{
Chronic pancreatitis associated acute respiratory
} \section{failure}

\begin{abstract}
Pancreatitis is a condition characterized by parenchymal inflammation of the pancreas, which is often associated with lung injury due to low level of oxygen and the condition is termed as (APALI). Clinical reports indicated that $\sim 20 \%$ to $50 \%$ of patients from low oxygen levels in blood with acute respiratory distress syndrome (ARDS). ARDS is a severe form of acute lung injury (ALI), a pulmonary disease with impaired airflow making patients difficult to breathe. ALI is frequently observed in patients with severe acute pancreatitis. Approximately one third of severe pancreatitis patients develop acute lung injury and acute respiratory distress syndrome that account for $60 \%$ of all deaths within the first week. The major causes of ALI and ARDS are sepsis, trauma, aspiration, multiple blood transfusion, and most importantly acute pancreatitis. The molecular mechanisms of ALI and ARDS are still not well explored, but available reports indicate the involvement of several proinflammatory mediators including cytokines (TNF- $\alpha$, IL-1 $\beta$, IL-6) and chemokines like interleukin-8 (IL-8) and macrophage inhibitory factor (MIF), as well as macrophage polarization regulating the migration and pulmonary infiltration of neutrophils into the pulmonary interstitial tissue, causing injury to the pulmonary parenchyma. Acute lung injury and acute respiratory distress syndrome in acute pancreatitis remains an unsolved issue and needs more research and resources to develop effective treatments and therapies. However, recent efforts have tested several molecules in an experimental model and showed promising results as a treatment option. The current review summarized the mechanism that is operational in pancreatitis-associated acute respiratory failure and respiratory distress syndrome in patients and current treatment options.
\end{abstract}

Keywords: acute lung injury, acute respiratory distress syndrome, acute pancreatitis, neutrophils, il-1 $\beta$, kynurenine 3-monooxygenase
Volume 5 Issue 2 - 2017

\author{
Anil Mishra,' Murli Manohar,' Alok K Verma,' \\ Sathisha Upparahalli Venkateshaiah,' Nathan \\ L Manders ${ }^{2}$ \\ Department of Medicine and Section of Pulmonary Diseases, \\ Tulane Eosinophilic Disorder Center,Tulane University School \\ of Medicine, USA \\ ${ }^{2}$ CELT Research Program, Tulane University, USA
}

Correspondence: Anil Mishra, Department of Medicine, Pulmonary Diseases, Tulane Eosinophilic Disorder Center,Tulane University School of Medicine, New Orleans, LA 70 I I2, USA, Tel (504)988-3840, Fax (504)988-0647,

Emailamishra@tulane.edu

Received: January 0I, 1970 | Published: February 08, 2017
Abbreviations: ALI, acute lung injury; ARDS, acute respiratory distress syndrome; IL-8, interleukin-8; JAM-C, junctional adhesion molecule-C; rTEM, reverse trans-endothelial migration; NETs, neutrophil extracellular traps; HMGB1, high mobility group box 1; IRF5, interferon regulatory factor 5; SAP, severe acute pancreatitis; KMO, kynurenine 3-monooxygenase

\section{Introduction}

Acute respiratory failure is a pulmonary disorder with impaired airflow, and it is difficult to breathe primarily due to the narrowing of the airways, termed airflow obstruction. ${ }^{1,2}$ Acute respiratory failure comprises both acute lung injury (ALI) and acute respiratory distress syndrome (ARDS). The most severe form of ALI, acute respiratory distress syndrome (ARDS), ${ }^{3}$ is frequently observed in patients with severe acute pancreatitis. ${ }^{4-6}$ ALI and ARDS can be distinguished on the basis of arterial oxygen pressure and inspired oxygen concentration ratio $(\mathrm{PaO} 2 / \mathrm{FiO} 2)$; ALI is defined as $(\mathrm{PaO} 2 /$ $\mathrm{FiO} 2) \leq 300 \mathrm{mmHg}$, whereas ARDS is $\mathrm{PaO} 2 / \mathrm{FiO} 2) \leq 200 \mathrm{mmHg} .{ }^{2,5}$ Pancreatitis is inflammation of the parenchyma of the pancreas in which acinar cell injury happens due to either poor dietary habits or mutation in the trypsinogen gene, and subsequently an accumulation of large numbers of inflammatory cells results such as neutrophils, eosinophils, mast cells, monocytes, and macrophages. ${ }^{7}$ In the initial phase of pancreatitis, the acute inflammatory responses that take place in the pancreas also affect pulmonary organ systems. ALI and ARDS frequently occur in severely affected acute pancreatitis patients, although the exact incidence in acute pancreatitis is unknown and there is a lack of complete understanding of the underlying mechanisms of ALI and ARDS in acute pancreatitis. Acute lung failure is the main contributing factor to early death in patients with severe acute pancreatitis due to single or multiple organ failure. ${ }^{8,9}$ Approximately one third of severe pancreatitis patients develops acute lung injury (ALI) or acute respiratory distress syndrome (ARDS), resulting in major health problems and accounting for approximately $60 \%$ of deaths from pancreatitis in the developed countries. ${ }^{10}$ The annual occurrence of ALI in the US is 190,000, with 74,500 deaths per year. ${ }^{1,2}$ Reports indicate that ALI is the consequence of systemic inflammatory responses that control the magnitude of pulmonary injury. ${ }^{6,10}$

\section{Mechanism of ALI and ARDS in pancreatitis}

The major causes of ALI and ARDS include sepsis, trauma, aspiration, multiple blood transfusions, and most importantly acute pancreatitis. ${ }^{1}$ There are two major phases known in the induction of ALI and ARDS. Phase (I) is an exudative phase, in which several events take place such as diffuse alveolar damage, type I pneumocyte necrosis, and an influx of inflammatory cells and fluid into the pulmonary interstitium. Phase (II) is a fibro-proliferative phase that is mainly characterized by repair of lung, type II pneumocyte hyperplasia,

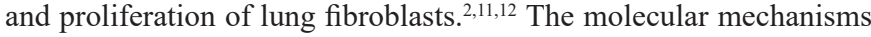
of ALI and ARDS is still not well explored, but available reports indicate the involvement of several pro-inflammatory mediators including cytokines (TNF- $\alpha$, IL-1 $\beta$, IL-6) $)^{2,13,14}$ and chemokines like interleukin-8 (IL-8) and macrophage inhibitory factor (MIF) $)^{2,15}$ as well as macrophage polarization regulating the migration and pulmonary infiltration of neutrophils into the pulmonary interstitial tissue, causing injury to the pulmonary parenchyma. ${ }^{16}$ A summarized mechanistic possible pathway involved in ALI is shown as a diagrammatic representation in Figure 1. 


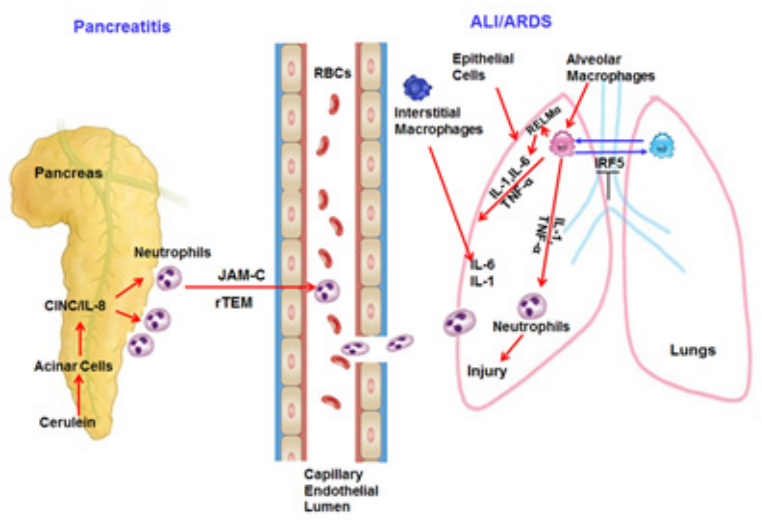

Figure I Mechanistic pathway involved in the induction of pancreatitisassociated acute respiratory syndrome.

Neutrophils are the main immune cells primarily recruited to the sites of pancreatic inflammation. Neutrophils are also considered to play a key role in the progression of lung inflammation. ${ }^{2}$ Several experimental models of pancreatitis have been used to study the role of neutrophils in lung inflammation. Improved survival rates and significantly decreased histological changes in rats were observed when antibody-depletion of peripheral neutrophils and blockage of neutrophil adherence functions using anti-CD18 antibodies were conducted. ${ }^{17}$ Further reports indicate administration of antineutrophil serum significantly reduces microvascular permeability and myeloperoxidase increase in the lungs of both experimental dietinduced as well as cerulein-induced pancreatitis model. ${ }^{18,19}$ A very recent report indicates that during $\mathrm{AP}$, neutrophils that are recruited to the pancreas may reverse migrate back into the circulation and further contribute to ALI. These reverse migrated neutrophils during ALI are regulated by Junctional adhesion molecule-C (JAM-C), and this process is termed reverse trans-endothelial migration (rTEM) of neutrophils. ${ }^{20}$ Another interesting report examined the process that neutrophils use to eliminate invading microorganisms by expelling nuclear DNA and histones to form extracellular weblike structures called neutrophil extracellular traps (NETs). These NETs were depleted by administration of DNase I enzyme to mice and revealed decreased neutrophil infiltration, reduced tissue damage and inflammation in the pancreas and lung, and decreased levels of blood amylase, macrophage inflammatory protein-2, interleukin 6 , and high-mobility groups protein- ${ }^{21}$ Further, downregulation of high mobility group box 1 (HMGB1) protects against the development of acute lung injury after severe acute pancreatitis via regulation of nuclear translocation of NF- $\kappa \mathrm{B} .{ }^{22}$

\section{Macrophages}

Migration of neutrophils into the lungs is a characteristic feature of ALI; however, native macrophages that reside in the pulmonary alveoli and interstitium are also thought to play a crucial role in the pathogenesis of ALI during severe acute pancreatitis. ${ }^{23-25}$ The cross talk of neutrophils and macrophages results in the release of a number of cytokines and chemokines that further progress to ALI. In general, there are two known types of macrophages, M1 and M2. M1 macrophages, also known as classically activated macrophages, are crucial effector cells and eliminate micro-organisms. ${ }^{26}$ M2 macrophages, also known as alternatively activated macrophages, are mainly involved in regulating the resolution of inflammatory responses. ${ }^{27}$ In the lung, these macrophages are further sub-grouped into alveolar, pleural, interstitial and intravascular macrophages. ${ }^{23-25}$
Krausgruber et al., ${ }^{28}$ have shown that Interferon regulatory factor 5 (IRF5) is a major factor in defining macrophage polarization and is a main regulator of polarization of pro-inflammatory M1 macrophages, inducing the expression of pro-inflammatory cytokines which downregulate transcription of anti-inflammatory cytokines ${ }^{28}$ A recent study has indicated that downregulation of IRF5 can reverse the pancreatitis-induced activation of lung macrophages from M1 phenotype to M2 phenotype in SAP associated with ALI. Therefore, IRF5 is an attractive target for therapy of severe acute pancreatitis (SAP) associated with ALI. However, the molecular mechanisms of IRF5 in macrophage polarization should be further studied. ${ }^{29}$ The activated macrophage-derived cytokine ResistinLike Molecule- $\alpha$ (RELM- $\alpha$ ) is an important cytokine that promotes inflammation in respective tissues. RELM- $\alpha$ belongs to a family of small cysteine-rich secreted proteins ${ }^{30}$ that negatively regulate lung inflammation via limiting the pathogenesis of Th2 cytokine-mediated pulmonary inflammation, in part through the regulation of CD4-T cell responses ${ }^{31}$ We earlier showed that induced RELM- $\alpha$ promotes lung and esophageal remodeling including fibrosis. ${ }^{32-34}$ It has been shown that RELM- $\alpha$ promotes lung injury in a rodent model of acute pancreatitis via activation of PI-3K/Akt-NF- $\mathrm{KB}$ pathway via inducing inflammatory cytokines like interleukin (IL)-1 $\beta$, IL-6, IL-8, tumor necrosis factor- $\alpha$, and serum C-reactive protein. ${ }^{35}$ In the absence of RELM $\alpha$, there is improved expression of proliferative cellular nuclear antigen, Bcl-2, zonal occludin-1 and Claudin-1 in lung tissue in the rat model of severe acute pancreatitis. ${ }^{35}$ These reports indicate that RELM- $\alpha$ has an important role in promoting lung injury severity in SAP by enhancing inflammatory cytokines.

\section{Cytokines and chemokines}

Several cytokines and chemokines such as TNF- $\alpha$, IL-1 $\beta$, IL-6, IL-8, MIF, and MCP-1 play crucial roles in acute pancreatitis-induced lung inflammation. ${ }^{15}$ TNF- $\alpha$ is a pleiotropic cytokine that acts as a key regulator of inflammation ${ }^{36-37}$ and is secreted by pancreatic acinar cells after an inflammatory trigger. ${ }^{38-41}$ Several reports have shown TNF- $\alpha$ plays an essential role in the pathogenesis of acute pancreatitis and contributes inflammatory responses to disease pathogenesis. ${ }^{38-40,42}$ TNF- $\alpha$ activates neutrophils that cause lung inflammation and dysfunction to other distal organs. ${ }^{43}$ Down-regulation of TNFassociated factor 6 (TRAF-6) is associated with progression of acute pancreatitis complicating lung injury in mice. ${ }^{13}$ IL-1 $\beta$ is also believed to play a role in the pathogenesis of pancreatitis associated lung inflammation. Mice lacking IL-1 $\beta$ converting enzyme (ICE/ caspases 1) have impaired IL-1 $\beta$ secretion and failed to develop acute pancreatitis and associated lung injury and have dramatic survival benefits. ${ }^{44}$ Interestingly, IL-1 receptor knockout and TNF- $\alpha$ receptor knockout mice failed to develop lung injury as compare to wildtype animals. ${ }^{14}$ IL-6 is a very important pro-inflammatory cytokine involved in inflammation and immune responses ${ }^{45}$ and is known to be elevated in the serum during acute pancreatitis as compared to normal individuals. ${ }^{46,47}$ CINC/IL-8 (Cytokine-induced neutrophil chemoattractant CINC in mice and analogous to Interleukin- 8 in human) is a major chemoattractant of neutrophils in the lungs and its increased levels have been found in the lungs of patients with ARDS. The use of neutralizing anti-CINC antibody in a ceruleininduced experimental model of acute pancreatitis revealed protective effects in the lungs. ${ }^{48}$ Macrophage migration inhibitory factor (MIF) is released by $\mathrm{T}$ lymphocytes, monocytes, macrophages and epithelial cells and is a pro-inflammatory cytokine and an important regulator of innate immunity. ${ }^{49}$ MIF mediates its pro-inflammatory activities by Toll-like receptor 4 (TLR4), resulting in the production of many pro-inflammatory cytokines, such as IL-6, IL-1 $\beta$, IL-8, TNF- $\alpha .{ }^{50}$ MIF 
is evolving as a critical key molecule in the pathogenesis of acute pancreatitis and pre-treatment with anti-MIF antibodies improved the survival of rats with AP. ${ }^{51}$ Serum MIF levels are also found to be significantly higher in severe AP than in mild AP or in healthy controls. ${ }^{51}$ The presence of MIF in the alveolar airspaces of patients with ARDS suggests that MIF may act as a mediator sustaining the pulmonary inflammatory response in ARDS, and that an anti-MIF strategy may represent a novel therapeutic approach in inflammatory diseases such as ARDS. ${ }^{52}$ IL-22, a member of the IL- 10 cytokine family, showed anti-inflammatory properties like IL-10 and protects mice from acute pancreatitis induced by cerulein and by a choline-deficient diet supplemented with DL-ethionine (CDE) ${ }^{53} \mathrm{~A}$ similar protection by IL-22 is also observed in severe acute pancreatitis (SAP)-associated lung injury induced by L-arginine in an experimental model. IL-22 mediates its protective effect by increasing the expression level of anti-apoptosis genes, such as Bcl-2 and Bcl-xL, through the STAT3 signaling pathway. ${ }^{54}$

\section{Current approaches for treatment of severe acute pancreatitis (SAP) associated acute lung injury (ALI)}

Acute lung injury and acute respiratory distress syndrome in acute pancreatitis remains an unsolved issue and needs more research and resources to develop effective treatment therapy. However, recent efforts have tested several molecules in experimental models and showed promising results as treatment options. The significance of some of these molecules we present in this review.

\section{Kynurenine 3-monooxygenase inhibitors}

Kynurenine 3-monooxygenase (KMO) is an enzyme of tryptophan metabolismand catalyzes the conversion ofL-Kynurenine to 3-hydroxyL-Kynurenine which causes oxidative stress ${ }^{55}$ and induces apoptosis. ${ }^{56}$ $\mathrm{KMO}$ is an important branch point in the kynurenine pathway and an attractive drug target for immunological, neurodegenerative, and neuro-inflammatory diseases. ${ }^{57}$ Kynurenine is reported to contribute to acute lung injury in an acute pancreatitis model of rats and increased levels of Kynurenine have been reported in the blood of severe acute pancreatitis patients. ${ }^{58}$ Interestingly, a very recent study by Mole and coworkers has generated global KMO knockout mice which are protected against extra pancreatic tissue injury to lung, kidney, and liver in experimental acute pancreatitis-multiple organ dysfunction syndrome (AP-MODS). Additionally, treatment with GSK180, a specific and potent KMO inhibitor, resulted in regulation of metabolites of the kynurenine pathway and showed promising therapeutic protection against AP-MODS in a rat model of AP. ${ }^{59}$ These findings indicate KMO inhibition may serve as a novel therapeutic strategy in the treatment of AP-MODS (including lung injury) and provide a new avenue for drug discovery to cure the pathogenesis of AP-induced multiple organ failure.

\section{Caspase inhibitors}

Pulmonary apoptosis is an important pathogenic mechanism of acute lung injury during acute pancreatitis. Caspase-1/ICE is a member of the family of cysteine proteases called caspases that play major roles in regulating apoptosis. ICE generates active IL- $1 \beta$ from its precursor and induces the activation of IL-6 and IL-18. Therefore, activation of Caspase- 1 and thus overproduction of IL-1 $\beta$ and IL18 in the lungs plays an important role during ALI and ARDS in SAP. Interestingly, ICE inhibitors (such as Ac-Tyr-Val-Ala-Asp-2,6dimethyl benzoyl oxymethyl ketone) are effective for the treatment of ALI in experimental SAP. ${ }^{60}$ A recent report showed the caspase inhibitor zVAD-fmk to protect against acute pancreatitis-associated lung injury via inhibiting the levels of MPO, TNF- $\alpha$, IL- $1 \beta$, and caspase- $3 .{ }^{61}$ These findings indicate that apoptosis plays a critical role in acute pancreatitis-associated lung injury, and inhibition of caspase activity may serve as a new therapeutic approach for the treatment of acute pancreatitis-associated lung injury, however the detailed mechanism need to be explored further.

\section{Resolvins}

Resolvins are new group anti-inflammatory derivatives of lipids such as eicosapentaenoic acid and docosahexaenoic acid with two chemically unique structural forms, the E-series and D-series of resolvins. E-series member resolvin E1 reduces inflammation in vivo and blocks human neutrophil transendothelial migration. ${ }^{62}$ Resolvin D1 (RvD1) as an endogenous anti-inflammatory lipid mediator has been confirmed to protect against many inflammatory diseases. ${ }^{63}$ Resolvin D1 protects against inflammation in experimental acute pancreatitis and associated lung injury. ${ }^{64} \mathrm{RvD} 1$ can improve injury of the pancreas and lung by exerting anti-inflammatory effects through the inhibition of NF- $\mathrm{kB}$ activation in experimental acute pancreatitis, with more notable protective effects in severe acute pancreatitis. This recent finding indicates that RvD1 may constitute a novel therapeutic strategy in the management of severe acute pancreatitis. ${ }^{65}$

\section{Phytocompounds used for treatment of SAP associated lung inflammation}

Several phytochemicals have been reported with various beneficial effects and they have been utilized in a variety of complementary and alternative approaches or as natural remedies over the past decades. The most beneficial aspect of these phytochemicals is the lack of side effects. Therefore, in search of more treatment options for SAP associated ALI, researchers have tested several phytochemicals such as resveratrol ${ }^{65}$ and apigenin ${ }^{66}$ in experimental models and these phytochemicals have revealed promising protective potential for the treatment of SAP associated ALI.

\section{Resveratrol}

Resveratrol (3,5,4'-trihydroxy-trans-stilbene) is a stilbenoid, a type of natural phenol, and a phytoalexin and is mainly found in grapes. Resveratrol has anti-inflammatory and anti-oxidant properties and its beneficial effect on severe acute pancreatitis has been reported in an experimental model..$^{67,68}$ Resveratrol is converted to dihydroresveratrol by gut microbes when taken orally. ${ }^{69}$ Interestingly, dihydroresveratrol reduces pancreatic oxidative damage during experimental pancreatitis. $^{70}$ In addition to this, dihydro-resveratrol showed protective effects against lung injury in rats with cerulein-induced acute pancreatitis. ${ }^{65}$ These findings indicate that dihydro-resveratrol may hold a promising therapeutic potential for the treatment of lung injury during acute pancreatitis.

\section{Apigenin}

Apigenin (4',5,7-trihydroxyflavone), a natural product belonging to the flavone class, is found in many plants. The role of apigenin in an experimental model of acute pancreatitis has been tested and results indicate oral apigenin administration in rats, following experimentallyinduced pancreatitis, seems to protect the pancreatic tissue. Thus, apigenin administration to humans could potentially ameliorate damage to the pancreas. ${ }^{71}$ Apigenin is also effective in experimental chronic pancreatitis where it inhibits the activity of pancreatic stellate cells. ${ }^{72}$ Furthermore, apigenin attenuates lung inflammation in experimentally induced acute pancreatitis. Results indicate improvement of lung tissue damage following apigenin administration. Apigenin reduces most histopathological alterations of the pulmonary tissue and decreases MPO and TNF- $\alpha$ activity. ${ }^{66}$ 


\section{Omega -3 fatty acids ( $\omega-3$ FA)}

$\omega-3 F A$, which are major components of fish oil-supplemented parenteral nutrition, offers a potential positive effect on the antiinflammatory response. Several animal studies have also shown that $\omega$-3FA can have an anti-inflammatory role in pancreatitis. ${ }^{73,74} \mathrm{~A}$ recent study indicates a protective effect of $\omega$-3FA during the initial stage of SAP; $\omega$-3FA can efficiently lower the inflammatory response and reduce lung injury by triggering the toll-like receptor 4 / nuclear factor

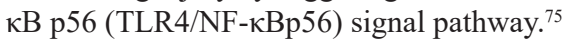

\section{Conclusion}

Taken together, the current review provides a summarized understanding of acute respiratory disorders such as ALI and ARDS during acute pancreatitis and remains an unsolved problem that needs more research and attention of investigators. Currently, there is lack of complete understanding of the underlying pathophysiological mechanisms of ALI/ARDS and vigorous effort is required to explore better treatment options. We provided details of involvement of key cells, pro-inflammatory cytokines and chemokines, and available treatment options to protect against lung injury and acute pancreatitis. The information provided in this review might be useful for investigators to focus on as we describe the cells and their associated mediators that might be helpful for future strategies for diagnostic and therapeutic interventions in the treatment of pancreatitis associated ALI/ARDS

\section{Conflicts of interest}

Authors have declared no conflict of interest.

\section{Acknowledgments}

Dr. Mishra is the endowed Schlieder Chair; therefore, authors thank Edward G. Schlieder Educational Foundation for the support. Additionally, Dr. Mishra's NIH R01 AI080581 grant funding supports the authors.

\section{Funding}

None.

\section{References}

1. Tsushima K, King LS, Aggarwal NR, et al. Acute lung injury review. Intern Med. 2009;48(9):621-630.

2. Akbarshahi H, Rosendahl AH, Westergren-Thorsson G, et al. Acute lung injury in acute pancreatitis--awaiting the big leap. Respir Med 2012;106(9):1199-1210.

3. Karnik ND, Gupta AV. Predicting Survival in ARDS. J Assoc Physicians India. 2015;63(11):11-13.

4. Milberg JA, Davis DR, Steinberg KP, et al. Improved survival of patients with acute respiratory distress syndrome (ARDS): 1983-1993. JAMA . 1995;273(4):306-309.

5. Bernard GR, Artigas A, Brigham KL, et al. The American-European Consensus Conference on ARDS. Definitions, mechanisms, relevant outcomes, and clinical trial coordination. Am J Respir Crit Care Med. 1994;149(3 pt 1):818-824.

6. Zhao X, Andersson R, Wang X, et al. Acute pancreatitis-associated lung injury: pathophysiological mechanisms and potential future therapies. Scand J Gastroenterol . 2002;37(12):1351-1358

7. Manohar M, VermaAK, Venkateshaiah SU, et al. Pathogenic mechanisms of pancreatitis. World J Gastrointest Pharmacol Ther . 2016;8(1):10-25.
8. Jacobs ML, Daggett WM, Civette JM, et al. Acute pancreatitis: analysis of factors influencing survival. Ann Surg.1977;185(1):43-51.

9. Pastor CM, Matthay MA, Frossard JL. Pancreatitis-associated acute lung injury: new insights. Chest . 2003;124(6):2341-2351.

10. Shields CJ, Winter DC, Redmond HP. Lung injury in acute pancreatitis: mechanisms, prevention, and therapy. Curr Opin Crit Care. 2002;8(2):158-163.

11. Tomashefski JF. Pulmonary pathology of the adult respiratory distress syndrome. Clin Chest Med. 1990;11(4):593-619.

12. Tomashefski, JF. Pulmonary pathology of acute respiratory distress syndrome. Clin Chest Med. 2000;21(3):435-466.

13. Zhou X, Li Y, Ding J, et al. Down-regulation of tumor necrosis factor-associated factor 6 is associated with progression of acute pancreatitis complicating lung injury in mice. Tohoku $J$ Exp Med. 2009;217(4):279-285.

14. Denham W, Yang J, Norman J. Evidence for an unknown component of pancreatic ascites that induces adult respiratory distress syndrome through an interleukin-1 and tumor necrosis factor-dependent mechanism. Surgery. 1997;122(2):295-301.

15. Pastor CM, Frossard JL. Are genetically modified mice useful for the understanding of acute pancreatitis? FASEB J. 2001;15(6):893-897.

16. Neumann B, Zantl N, Veihelmann A, et al. Mechanisms of acute inflammatory lung injury induced by abdominal sepsis. Int Immunol 1999;11(2):217-227.

17. Inoue $\mathrm{S}$, Nakao $\mathrm{A}$, Kishimoto $\mathrm{W}$, et al. Anti-neutrophil antibody attenuates the severity of acute lung injury in rats with experimental acute pancreatitis. Arch Surg. 1995;130(1):93-98.

18. Guice KS, Oldham KT, Caty MG, et al. Neutrophil-dependent, oxygenradical mediated lung injury associated with acute pancreatitis. Ann Surg . 1989;210(6):740-747.

19. Bhatia M, Saluja AK, Hofbauer B, et al. The effects of neutrophil depletion on a completely noninvasive model of acute pancreatitisassociated lung injury. Int J Pancreatol. 1998;24(2):77-83.

20. Wu D, Zeng Y, Fan Y, et al. Reverse-migrated neutrophils regulated by JAM-C are involved in acute pancreatitis-associated lung injury. Sci Rep. 2016;6:20545.

21. Merza M, Hartman H, Rahman M, et al. Neutrophil Extracellular Traps Induce Trypsin Activation, Inflammation, and Tissue Damage in Mice With Severe Acute Pancreatitis. Gastroenterology. 2015;149(7):1920-1931.

22. Luan ZG, Zhang XJ, Yin XH, et al. Downregulation of HMGB1 protects against the development of acute lung injury after severe acute pancreatitis. Immunobiology. 2013;218(10):1261-1270.

23. Gea-Sorli S, Guillamat R, Serrano-Mollar A, et al. Activation of lung macrophage subpopulations in experimental acute pancreatitis. $J$ Pathol 2011;223(3):417-424.

24. Xu XW, Yang XM, Bai YH, et al. Treatment with ginkgo biloba extract protects rats against acute pancreatitis-associated lung injury by modulating alveolar macrophage. Prz Gastroenterol . 2014;9(1):43-48.

25. Xue P, Guo J, Yang XN, et al. Changes of neuronal acetylcholine receptor alpha 7 of peritoneal macrophage in experimental acute pancreatitis treated by Chaiqin Chengqi Decoction. Chin J Integr Med. 2014:20(10):770-775.

26. Mantovani A, Biswas SK, Galdiero MR, et al. Macrophage plasticity and polarization in tissue repair and remodelling. $J$ Pathol. 2013;229(2):176-85.

27. Mosser DM, Edwards JP. Exploring the full spectrum of macrophage activation. Nat Rev Immunol. 2008;8(12):958-969. 
28. Krausgruber T, Katrina Blazek, Tim Smallie, et al. Nat Immunol. 2011;12:231-238

29. Sun K, He SB, Qu JG, et al. IRF5 regulates lung macrophages M2 polarization during severe acute pancreatitis in vitro. World $J$ Gastroenterol . 2016;22(42):9368-9377.

30. Steppan CM, Brown EJ, Wright CM, et al. A family of tissue-specific resistin-like molecules. Proc Natl Acad Sci U S A. 2001;98(2):502-506.

31. Nair MG, Du Y, Perrigoue JG, et al. Alternatively activated macrophagederived RELM- $\{$ alpha $\}$ is a negative regulator of type 2 inflammation in the lung. J Exp Med . 2009;206(4):937-952.

32. Hogan SP, Seidu L, Blanchard C, et al. Resistin-like molecule beta regulates innate colonic function: barrier integrity and inflammation susceptibility. J Allergy Clin Immunol. 2006;118(1):257-268.

33. Mishra A, Wang M, Schlotman J, et al. Resistin-like molecule-beta is an allergen-induced cytokine with inflammatory and remodeling activity in the murine lung. Am J Physiol Lung Cell Mol Physiol. 2007;293(2):305-313.

34. Mavi P, Niranjan R, Dutt $P$, et al. Allergen-induced resistin-like molecule-alpha promotes esophageal epithelial cell hyperplasia in eosinophilic esophagitis. Am J Physiol Gastrointest Liver Physiol . 2014;307(5):499-507.

35. Wang WY, Chen Y, Su X, et al. Resistin-Like Molecule-alpha Causes Lung Injury in Rats with Acute Pancreatitis by Activating the PI-3K Akt-NF-kappaB Pathway and Promoting Inflammatory Cytokine Release. Curr Mol Med. 2016;16(7):677-687.

36. Zhang JM, An J. Cytokines, inflammation, and pain. Int Anesthesiol Clin 2007;45(2):27-37.

37. Esposito E, Cuzzocrea S. TNF-alpha as a therapeutic target in inflammatory diseases, ischemia-reperfusion injury and trauma. Curr Med Chem. 2009;16(24):3152-3167.

38. Malleo G, Mazzon E, Siriwardena AK, et al. Role of tumor necrosis factor-alpha in acute pancreatitis: from biological basis to clinical evidence. Shock. 2007;28(2):130-140.

39. Malleo G, Mazzon E, Siriwardena AK, et al. TNF-alpha as a therapeutic target in acute pancreatitis--lessons from experimental models. ScientificWorldJournal . 2007;7:431-448.

40. Norman JG, Fink GW, Franz MG. Acute pancreatitis induces intrapancreatic tumor necrosis factor gene expression. Arch Surg. 1995;130(9):966-970.

41. Hohmann HP, Remy R, Brockhaus M, et al. Two different cell types have different major receptors for human tumor necrosis factor (TNF alpha). J Biol Chem. 1989;264(25):14927-14934.

42. Schäfer C, Tietz AB, Göke B. Pathophysiology of acute experimental pancreatitis: lessons from genetically engineered animal models and new molecular approaches. Digestion. 2005; 71(3):162-172.

43. Norman JG, Fink GW, Denham W, et al. Tissue-specific cytokine production during experimental acute pancreatitis. A probable mechanism for distant organ dysfunction. Dig Dis Sci. 1997; 42(8):1783-1788.

44. Norman J, Yang J, Fink G, et al. Severity and mortality of experimental pancreatitis are dependent on interleukin-1 converting enzyme (ICE). $J$ Interferon Cytokine Res. 1997;17(2):113-118.

45. Lesina $M$, Wörmann SM, Neuhöfer $P$, et al. Interleukin-6 in inflammatory and malignant diseases of the pancreas. Semin Immunol 2014;26(1):80-87.

46. Berney T, Gasche Y, Robert J, et al. Serum profiles of interleukin-6, interleukin-8, and interleukin-10 in patients with severe and mild acute pancreatitis. Pancreas. 1999;18(4):371-377.

47. Hansen M, Nielsen AR, Vilsbøll T, et al. Increased levels of YKL-40 and interleukin 6 in patients with chronic pancreatitis and secondary diabetes. Pancreas . 2012;41(8):1316-1318.
48. Bhatia M, Brady M, Zagorski J, et al. Treatment with neutralising antibody against cytokine induced neutrophil chemoattractant (CINC) protects rats against acute pancreatitis associated lung injury. Gut. 2000;47(6):838-844.

49. Funamizu N, Hu C, Lacy C, et al. Macrophage migration inhibitory factor induces epithelial to mesenchymal transition, enhances tumor aggressiveness and predicts clinical outcome in resected pancreatic ductal adenocarcinoma. Int J Cancer. 2013;132(4):785-794.

50. Calandra T, Roger T. Macrophage migration inhibitory factor: a regulator of innate immunity. Nat Rev Immunol . 2003;3(10):791-800.

51. Sakai Y, Masamune A, Satoh A, et al. Macrophage migration inhibitory factor is a critical mediator of severe acute pancreatitis. Gastroenterology. $2003 ; 124(3): 725-736$

52. Donnelly SC, Haslett C, Peter TR, et al. Regulatory role for macrophage migration inhibitory factor in acute respiratory distress syndrome. Nat Med. 1997;3(3):320-323.

53. Xue J, Nguyen DT, Habtezion A. Aryl hydrocarbon receptor regulates pancreatic IL-22 production and protects mice from acute pancreatitis. Gastroenterology. 2012;143(6):1670-1680.

54. Qiao YY, Liu XQ, Xu CQ, et al. Interleukin-22 ameliorates acute severe pancreatitis-associated lung injury in mice. World $J$ Gastroenterol . 2016;22(21):5023-5032.

55. Mole DJ, Olabi B, Robinson V, et al. Incidence of individual organ dysfunction in fatal acute pancreatitis: analysis of 1024 death records. HPB (Oxford). 2009;11(2):166-170.

56. Zwilling D, Huang SY, Sathyasaikumar KV, et al. Kynurenine 3-monooxygenase inhibition in blood ameliorates neurodegeneration. Cell. 2011;145(6): 863-874.

57. Smith JR, Jamie JF, Guillemin GJ. Kynurenine-3-monooxygenase: a review of structure, mechanism, and inhibitors. Drug Discov Today.2016;21(2):315-324.

58. Mole DJ, McFerran NV, Collett G, et al. Tryptophan catabolites in mesenteric lymph may contribute to pancreatitis-associated organ failure. Br J Surg . 2008;95(7):855-867.

59. Mole DJ, Webster SP, Uings I, et al. Kynurenine-3-monooxygenase inhibition prevents multiple organ failure in rodent models of acute pancreatitis. Nat Med. 2016;22(2):202-209.

60. Zhang XH, Zhu RM, Xu WA, et al. Therapeutic effects of caspase-1 inhibitors on acute lung injury in experimental severe acute pancreatitis. World J Gastroenterol . 2007;13(4):623-627.

61. Liu M, Shi L, Zou X, et al. Caspase inhibitor zVAD-fmk protects against acute pancreatitis-associated lung injury via inhibiting inflammation and apoptosis. Pancreatology. 2016;16(5):733-738.

62. Serhan CN, Clish CB, Brannon J, et al. Novel functional sets of lipidderived mediators with antiinflammatory actions generated from omega-3 fatty acids via cyclooxygenase 2-nonsteroidal antiinflammatory drugs and transcellular processing. J Exp Med. 2000;192(8):1197-204.

63. Serhan CN, Chiang N, Van Dyke TE. Resolving inflammation: dual anti-inflammatory and pro-resolution lipid mediators. Nat Rev Immunol. 2008;8(5):349-361.

64. Liu Y, Zhou D, Long FW, et al. Resolvin D1 protects against inflammation in experimental acute pancreatitis and associated lung injury. Am J Physiol Gastrointest Liver Physiol . 2016;310(5):G303-G309.

65. Sarwar S, Lin ZS, Ku CF, et al. Protective effect of dihydro-resveratrol against lung injury in rats with cerulein-induced acute pancreatitis. Planta Med. 2016;81(S 01):S1-S381.

66. Basios N, Lampropoulos P, Papalois A, et al. Apigenin Attenuates Inflammation in Experimentally Induced Acute Pancreatitis-Associated Lung Injury. J Invest Surg. 2016;29(3):121-127. 
67. Jha RK, Ma Q, Lei Z, Sha H. Resveratrol ameliorates the deleterious effect of severe acute pancreatitis. Cell Biochem Biophys. 2012;62(2):397-402.

68. Ma Q, Zhang M, Wang Z, et al. The beneficial effect of resveratrol on severe acute pancreatitis. Ann N Y Acad Sci. 2011;1215:96-102.

69. Wang D, Hang T, Wu C, et al. Identification of the major metabolites of resveratrol in rat urine by HPLC-MS/MS. J Chromatogr B Analyt Technol Biomed Life Sci. 2005;829(1-2):97-106.

70. Tsang SW, Guan YF, Wang J, et al. Inhibition of pancreatic oxidative damage by stilbene derivative dihydro-resveratrol: implication for treatment of acute pancreatitis. Sci Rep. 2016;6:22859.

71. Lampropoulos P, Lambropoulou M, Papalois A, et al. The role of apigenin in an experimental model of acute pancreatitis. J Surg Res. 2013;183(1):129-137.
72. Mrazek AA, Porro LJ, Bhatia V, et al. Apigenin inhibits pancreatic stellate cell activity in pancreatitis. J Surg Res. 2015;196(1):8-16.

73. Alhan E, Türkyilmaz S, Erçin C, et al. Effects of omega-3 fatty acids on acute necrotizing pancreatitis in rats. Eur Surg Res. 2006;38(3):314-321.

74. Foitzik T, Eibl G, Schneider P, et al. Omega-3 fatty acid supplementation increases anti-inflammatory cytokines and attenuates systemic disease sequelae in experimental pancreatitis. JPEN J Parenter Enteral Nutr. 2002;26(6):351-356.

75. Wang B, Wu XW, Guo MX, et al. Effects of $\omega-3$ fatty acids on toll-like receptor 4 and nuclear factor-kappaB p56 in lungs of rats with severe acute pancreatitis. World J Gastroenterol. 2016;22(44):9784-9793. 